\title{
Kostenmanagement in Supply Chains
}

\author{
Prof. Dr. Wolfgang Stölzle \\ Dr. Erik Hofmann \\ Florian Hofer \\ Universität St.Gallen, Schweiz \\ Logistik-Management 2005 \\ Sektion 3: Logistik-Controlling \\ Dresden \\ 05. Oktober 2005
}


Agenda

F. Hofer
Seite 2

1 Ausgangssituation

2 Überblick über bestehende Ansätze des unternehmensübergreifenden Kostenmanagements

3 Bezugsrahmen für ein unternehmensübergreifendes Kostenmanagement

4 Ausgangspunkt für und Anforderungen an die Elemente des Supply Chain Costing

5 Ausgewählte Instrumente für das Supply Chain Costing

6 Ausblick 
Auf Sachzielebene verfolgt die Supply Chain Management-Konzeption die Steigerung des Endkundennutzens, die Realisierung von Zeitvorteilen, die Verbesserung der Qualität sowie die Kostenreduktion. ${ }^{1}$

- Durchgängige Ausrichtung der Servicekomponenten an den Bedürfnissen der Endkunden

- Verkürzung von Marktbearbeitungszeiten, Reduzierung von Redundanzen

- Konzepte zur Steigerung der Produkt- und Prozessqualität

- Investitions-, Finanzierungs- und Bestandskosten sowie Kosten für Transport, Lagerung und Umschlag

Steuerung und Beeinflussung von Kosten kein expliziter Bestandteil der Supply Chain Management-Konzeption 
Kostenmanagement umfasst die Gesamtheit aller Steuerungsmassnahmen zur zielgerichteten Beeinflussung des Niveaus sowie der Struktur und Verläufe von Kosten, die sich auf Produkte, Prozesse und Produktionsfaktoren beziehen. ${ }^{1}$

- Instrumente des Kostenmanagement i.d.R. auf einzelne Unternehmen ausgerichtet

- Fehlende unternehmensübergreifende, netzwerkbezogene Analyse und Gestaltung der anfallenden Kosten

- Interorganisatorische Kostenbetrachtung zur Erreichung der Supply Chain Management-Ziele von Bedeutung

Systematische Integration des Kostenmanagements in ein unternehmensübergreifendes Konzept steht aus 
- Pohlen/LaLonde (1996):

- Wertmässige Abbildung des Material-, Waren- und Informationsflusses über mehrere Wertschöpfungsstufen (prozessorientierter Ansatz)

- Ermittlung der Gesamteffizienz der SC, Effizienzmessung einzelner Prozesse

- Messbarkeit der Kosten

- Cooper/Slagmulder (1999):

- „Interorganisational Cost Management"

- Koordination von Unternehmensaktivitäten zur Senkung von Kosten in Wertschöpfungsnetzwerken

- Nachgeordnete Thematisierung von Massnahmen zur Beeinflussung von Kostenstruktur, -verlauf und -niveau

- Seuring (2001):

- Differenzierung nach Einzelkosten, Prozesskosten, Transaktionskosten

- Effizientere Kostenbeeinflussung

- Höhere Kostentransparenz

Neben Messbarkeit und Differenzierung nach Kostenkategorien muss auch die Kostenbeeinflussung von Elementen der Supply Chain Management-

\section{Konzeption berücksichtigt werden!}




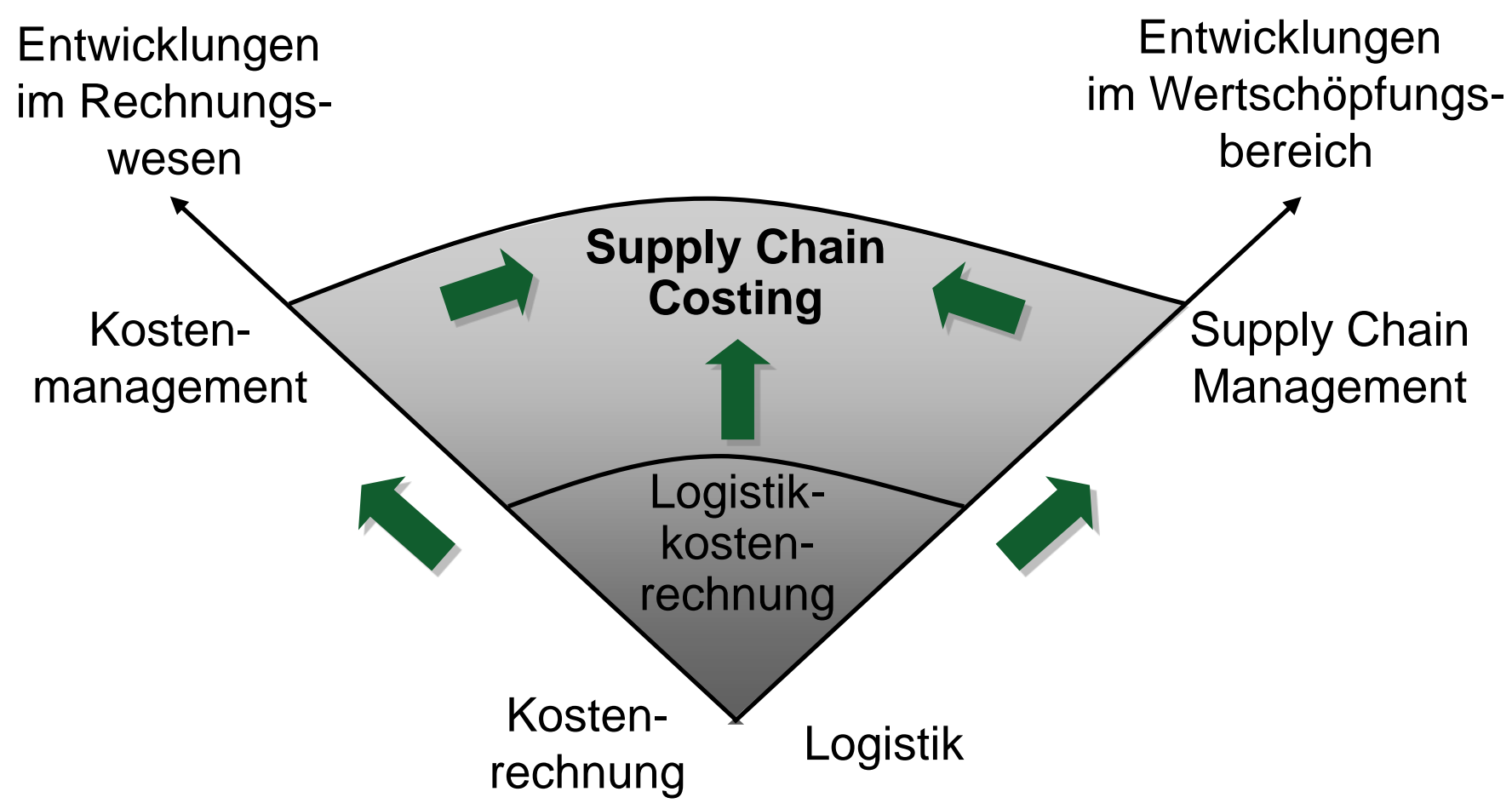

Supply Chain Costing erfährt Impulse aus den Entwicklungen im Rechnungswesen und im Wertschöpfungsbereich 
4 Kostenrechnung - Ausgangspunkt und Anforderungen im Hinblick auf das Supply Chain Costing

\section{Ausgangspunkt:}

- Bestehende interne Kostenerfassung und -verrechnung mit spezifisch ausgelegten Rechnungssystemen

- Gemeinkostenzuschlagsätze und Wahl der Bezugsgrössen sind Ergebnis rein unternehmensbezogener Kostenrechnung ${ }^{1}$

- Nicht-standardisierte Kosteninformationen sind mit unzureichender Güte der Kostenaussagen verbunden

\section{Anforderungen im Hinblick auf das Supply Chain Costing:}

- Verursachungsgerechte Kostenzuordnung über mehrere Unternehmen hinweg

- Abstimmungsmechanismen über Modalitäten der Kostenerfassung und -verrechnung

- „Kostenrechnungsinseln“ sind miteinander zu verknüpfen

- Netzwerkweite Harmonisierung von Kostenkategorien und -bezugsgrössen

- Abstimmung der Zurechnungsobjekte von Kosten zwischen den Supply ChainAkteuren 
4 Logistikkostenrechnung - Ausgangspunkt und Anforderungen im Hinblick auf das Supply Chain Costing

\section{Ausgangspunkt:}

- Ermittlung der Logistikkosten in Supply Chains von hoher Bedeutung für die Entscheidungsfindung

- Herausforderung der einheitlichen Verrechnung von Logistikkosten aufgrund spezifischer Kosteneinflussgrössen

- Anpassung unternehmensbezogener Kostenrechnungssysteme an die Besonderheiten von Logistikkosten ${ }^{1}$

\section{Anforderungen im Hinblick auf das Supply Chain Costing:}

- Detaillierungsgrad der unternehmensbezogenen Logistikkostenrechnung $\rightarrow$ „Differenzierung in die Tiefe“

- Ausdehnung auf vor- und/oder nachgelagerte Wertschöpfungsstufen $\rightarrow$ „Differenzierung in der Länge“

- Diffizile Zuordnung von Logistikkosten, die zwischen Supply Chain-Akteuren anfallen 
4 Supply Chain Management-Konzeption - Ausgangspunkt und Anforderungen im Hinblick auf das Supply Chain Costing

\section{Ausgangspunkt:}

- Beeinflussung von Kosten durch Supply Chain-Strukturveränderungen

- Gestaltung effizienter Geschäftsprozesse

- Einsatz von unternehmensübergreifenden Managementkonzepten (bspw. SCEM)

\section{Anforderungen im Hinblick auf das Supply Chain Costing:}

- Messung und Steuerung von Kosten auf strategischer und operativer Ebene

- Identifikation der Kostenwirkungen bei Prozessabweichungen

- Kostenmanagement zur Untermauerung unternehmensübergreifender Investitionsentscheidungen

- Kostenbeeinflussung aufgrund von Entscheidungen über

Managementkomponenten 
5 Ausgewählte Kostenmanagementinstrumente für das Supply Chain Costing

- Aktueller Entwicklungsstand SCC: eher methodisch-instrumenteller Ansatz

- Supply Chain Costing benötigt Methoden und Instrumente, die für konkrete Ziele eingesetzt werden

- Gestaltung in Anlehnung an das unternehmensindividuelle Kostenmanagement:

- Produktbezogene Betrachtung

$\rightarrow$ Lebenszyklusorientierte Kostenrechnung über mehrere SC-Akteure

- Prozessbezogene Betrachtung

$\rightarrow$ Unternehmensübergreifende Prozesskostenrechnung

- Produktionsfaktoren

$\rightarrow$ Total Cost of Ownership über mehrere SC-Akteure

(Fokus auf spezifische Investitionen relevanter Produktionsfaktoren) 
5 Beurteilung der Instrumente des Kostenmanagements in Supply Chains (I)

- Instrumente lassen sich in die konstitutiven Elemente des Supply Chain Managements sowie in die Gestaltungsfelder des Kostenmanagements einordnen

- Methodisch-instrumentelle Herangehensweise erlaubt keine überschneidungsfreie Berücksichtigung

Die vorgestellten Instrumente weisen spezifische Stärken und Schwächen im Hinblick auf das unternehmensübergreifende Kostenmanagement in

\section{Supply Chains auf}




\section{Beurteilung der Instrumente des Kostenmanagements in Supply Chains (II)}

\section{Lebenszyklusorientierte} Kostenrechnung

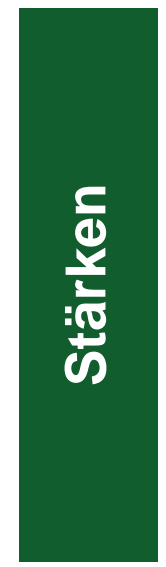

- Aufdeckung von

Schwachstellen im

Innovationsprozess

- Explizite Einbindung von SCAkteuren

- Berücksichtigung von Einzelund Gemeinkosten

- Unzureichende Transparenz von Kosten nach erfolgter Produkteinführung

- Operatives KM in SCs schwer realisierbar

- Definition „relevanter“ Kostenkategorien erschwert

- Akzeptanz bei SC-Akteuren eingeschränkt
Unternehmensübergreifende Prozesskostenrechnung

- Prozesstransparenz deckt Kosteneinsparungspotenziale auf

- Standardisierte Kostenkategorien und einheitliche Kostentreiber unterstützen Entscheidungsfindung

- Kostentransparenz erlaubt Kostenvergleich

- Diffizile Harmonisierung von Prozessdefinition

- Uneinheitliche Zuordnung von Prozesskosten auf Teil-/ Hauptprozesse

- Unterschiedliche Identifikation von Kostenkategorien und -treibern

\section{Total Cost of Ownership}

- Erhöhte Kostentransparenz unterstützt Gestaltungsentscheidungen der Geschäftsprozesse/Transaktionen

- Gemeinsame Analyse der Kostenstruktur unterstützt Kommunikation

- Verbesserte Planungsqualität

- Geringe Abbildungsfähigkeit einzelner Kostenkategorien

- Abstimmungsprobleme bzgl. Transaktionsabgrenzung, Kostenkategorien

- Keine Berücksichtigung von Bewertung und Modalitäten der Kostenkategorien 
- Methodisch-instrumentelle Betrachtung des unternehmensübergreifenden Kostenmanagements erforderlich

- Unmittelbare Anwendung bekannter Kostenmanagementinstrumente aufgrund unternehmensübergreifender Ausrichung erschwert

- Kombinierter Einsatz mehrerer Instrumente erfüllt nur bedingt Anforderungen des Kostenmanagements in Supply Chains

- Umfassende Konzeptualisierung des Kostenmanagements im Hinblick auf das Supply Chain Management noch nicht erarbeitet

- Forschungsbedarf besteht im Hinblick auf standardisierte Leistungs- und Kostenerfassung sowie unternehmensübergreifendes Prozessverständnis in Supply Chains 


\section{Ausgewählte Literatur}

Back-Hock, A. (1997): Produktlebenszyklusorientierte Ergebnisrechnung. In: Männel, W. (Hrsg.): Handbuch Kostenrechnung. Wiesbaden, 1997, S. 703-714.

Baden, A. (1997): Straegische Kostenrechnung. Einsatzmöglichkeiten und Grenzen. Wiesbaden 1997.

Cooper, M./Lambert, D.M./Pagh, J.D. (1997): Supply Chain Management: More than a New Name fpr Logistics. In: The International Journal of Logistics Management 8(1997)1, S. 1-14.

Cooper, R. (1988): The Rise of Activity-Based Costing - Part One: What is an Acitivity-Based Cost System? In: Journal of Cost Management, Summer (1988), S. 45-54.

Cooper, R./Slagmulder, R. (1999): Supply Chain Development for the Lean Enterprise - Interorganisational Cost Management. Portland 1999.

Dekker, H.C./van Goor, A.R. (2000): Supply Chain Management and Management Accounting: A Case Study of Activity-Based Costing. In: International Journal of Logistics: Research and Applications 3(2000)1, S. 41-52.

Ellram, L.M. (1995): Total Cost of Ownership. An analysis approach for purchasing. In: International Journal of Physical Distribution \& Logistics Management 25(1995)8, S. 4-23.

Elram, L.M./Siferd, S.P. (1998): Total Cost of Ownership: A Key Concept in Strategic Management. In: Journal of Business Logistics 19(1998)1, S. 55-84.

Kajüter, P. (2000): Proaktives Kostenmanagement. Konzeption und Realprofile. Wiesbaden 2000.

LaLonde, B.J./Pohlen, T.L. (1996): Issues in Supply Chain Costing. In: The International Journal of Logistics Management 7(1996)1, S. 1-12.

Seuring, S. (2001): Supply Chain Costing: Kostenmanagement in der Wertschöpfungskette mit Target Costing und Prozesskostenrechnung. München 2001.

Weber, J. (2002): Logistik- und Supply Chain Controlling. 5., aktual. u. völlig überarb. Auflage. Stutgart 2002. 


\section{Vielen Dank für Ihre Aufmerksamkeit}

Prof. Dr. Wolfgang Stölzle

Dr. Erik Hofmann

Florian Hofer

Universität St.Gallen

Dufourstrasse 40a

CH-9000 St.Gallen 